\title{
ABAKO/RAPCAL: A Flexible Computational Package to Perform Radiative Properties Calculations and Diagnostics in a Wide Range of Plasma Conditions
}

Cite as: AIP Conference Proceedings 1058, 331 (2008); https://

doi.org/10.1063/1.3026474

Published Online: 27 October 2008

R. Florido, R. Rodríguez, J. M. Gil, J. G. Rubiano, P. Martel, E. Mínguez, R. Sauvan, and R. Mancini

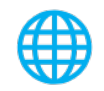

\section{Conference Proceedings}

\section{Get $30 \%$ off all print proceedings!}

Enter Promotion Code PDFBO at checkout 


\title{
ABAKO/RAPCAL: A Flexible Computational Package to Perform Radiative Properties Calculations and Diagnostics in a Wide Range of Plasma Conditions
}

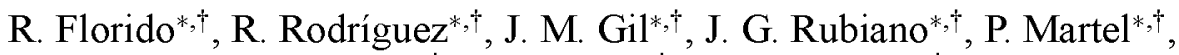 \\ E. Mínguez ${ }^{\dagger}$, P. Sauvan ${ }^{* * \dagger}$ and R. Mancini ${ }^{*}$ \\ ${ }^{*}$ Departamento de Física, Universidad de Las Palmas de Gran Canaria, 35017 Las Palmas de \\ Gran Canaria, Spain \\ ${ }^{\dagger}$ Instituto de Fusión Nuclear, Universidad Politécnica de Madrid, 28006 Madrid, Spain \\ ${ }^{* *}$ Departamento de Ingenieria Energética, UNED, Madrid, Spain \\ ${ }^{\ddagger}$ Department of Physics, University of Nevada, Reno, NV 89557, USA
}

\begin{abstract}
This work describes ABAKO/RAPCAL, a flexible computational package for the study of population kinetics and radiative properties of non-equilibrium plasmas in a wide range of physical conditions. The code was developed looking for an optimal compromise between accuracy and computational cost. ABAKO/RAPCAL assembles a set of simple analytical models which yield substantial savings of computer resources, but yet still providing good comparisons with more elaborated codes and experimental data. Here we present some results to show the ABAKO/RAPCAL capabilities to calculate the charge distribution and radiative properties of both low- and high-Z plasmas. Finally, an application for K-shell spectroscopic determination of the electron temperature and density of laser-produced plasmas is also shown.
\end{abstract}

Keywords: plasma atomic physics, NLTE plasmas, collisional-radiative, population distribution, radiative properties, spectroscopic diagnostics

PACS: $52.20 .-\mathrm{j}, 52.25 . \mathrm{Dg}, 52.25 . \mathrm{Os}$

\section{INTRODUCTION}

ABAKO/RAPCAL is a computational package composed by two numerical codes that has been recently developed at the University of Las Palmas de Gran Canaria. The first one named $\mathrm{ABAKO}[1]$ is a collisional-radiative $(\mathrm{CR})$ model that calculates atomic data and level populations for steady state plasmas. The second code is called RAPCAL [2] and it carries out the computation of radiative properties such as spectrally resolved and mean emissivities and opacities, intensities and radiative power losses. A remarkable characteristic of ABAKO/RAPCAL is its versatility. It can be applied to low-tohigh $\mathrm{Z}$ ions under a wide range of plasma conditions: coronal, local-thermodynamicequilibrium (LTE) or non-equilibrium (NLTE), optically thin or thick plasmas. Moreover, during the development of the computational package a special care was taken to achieve an optimal equilibrium between accuracy and computational cost. Next section describes the main features of ABAKO/RAPCAL. In the last section some relevant results are commented. 


\section{ABAKO/RAPCAL FEATURES}

ABAKO has a built-in atomic model to obtain all the data required for the determination of the level populations. This atomic model is framed into the relativistic detailedconfiguration-accounting approach and based on the use of analytical potentials developed by our group in several previous works [1]. It allows fast and accurate calculations of coarse magnitudes such as plasma average ionization and ion abundances. However, when a high accuracy is desired, more detailed atomic structure from databases or computational codes can be used. In particular, ABAKO has used atomic data provided by FAC code [3] in a detailed-level-accounting approach. Once the atomic data are available, $\mathrm{ABAKO}$ builds the $\mathrm{CR}$ matrix in which the following atomic processes are considered: spontaneous decay, collisional excitation and deexcitation, radiative recombination, collisional ionization and three-body recombination, electron capture and autoionization. The rate coefficients of the atomic processes are obtained using wellknown semiempirical analytical formulas. ABAKO uses an iterative method to carry out the inversion of the CR matrix. Furthermore, optically thick plasmas can be modeled. The escape factor formalism for basic geometries is used to take into account the boundbound opacity effects. For its part, RAPCAL computes spectrally resolved and mean opacities and emissivites, intensities, source functions and radiative power losses for optically thin and thick plasmas under LTE and NLTE conditions. Complete redistribution is assumed. A Voigt profile including natural, Doppler and electron-collisional broadenings is considered. The bound-bound spectra are obtained in the dipole approximation. The bound-free spectra can be obtained using the Kramer's formula for the cross section or by means of the distorted wave approach. The free-free spectra are calculated using the Kramer's approach and scattering Thomson is also considered.

\section{RESULTS}

A rigorous verification and validation procedure was carried out to assure the reliability of the ABAKO/RAPCAL results. The first stage was a study of internal consistency to check that ABAKO effectively converges to the Corona and LTE results in the opposite limits of low and high electron density, respectively [4]. In a second stage we compared the ABAKO results with other CR codes developed by diverse research groups in the atomic plasma physics community. The comparisons mainly came from computational tests proposed in the NLTE-3 and NLTE-4 workshops [5, 6] for different elements over a wide range of physical conditions. An example for the NLTE-4 carbon test case can be found in [1]. On the other hand, RAPCAL has been used to compute carbon emissivities, Rosseland and Planck mean opacities of carbon and aluminum for low and intermediate densities, as well as radiative power losses of krypton at low densities and several temperatures. Satisfactory comparisons were obtained in all these cases [2]. An example of comparison for an experimental aluminum transmission is shown in Fig. 1 (left). Additionally, good agreements with experimental data have been found in population calculations of higher-Z elements. For a Xe gas jet at $n_{i o n}=4.75 \times 10^{18} \mathrm{~cm}^{-3}$ and $T_{e}=415 \mathrm{eV}$ [7], the measured average ionization was 27.4 \pm 1.5 , while ABAKO gives $\bar{Z}=26.6$. For a Au EBIT experiment with $n_{e}=10^{12} \mathrm{~cm}^{-3}$ and $T_{e}=2500 \mathrm{eV}$ [8], 

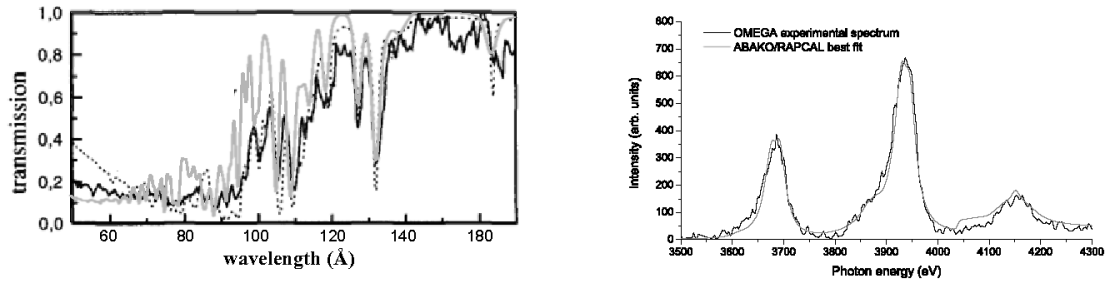

FIGURE 1. On the left it is shown the comparison of the experimental aluminum transmission spectrum (black solid) with OPAL (dotted) and RAPCAL (gray solid) [2]. On the right we show the ABAKO/RAPCAL best fit for an argon K-shell emission spectrum recorded in an implosion experiment performed at OMEGA [10]. The analysis gives $n_{e}=1.174 \times 10^{24} \mathrm{~cm}^{-3}$ and $T_{e}=1526 \mathrm{eV}$.

the measurement was $\bar{Z}=46.8 \pm 0.75$ and ABAKO gives $\bar{Z}=47.2$. Also for published Au data from foil with $n_{e}=6 \times 10^{20} \mathrm{~cm}^{-3}, T_{e}=2200 \mathrm{eV}$ and $\bar{Z}=49.3 \pm 0.5$ [9], the $\mathrm{ABAKO}$ result is $\bar{Z}=49.2$. In addition, $\mathrm{ABAKO} / \mathrm{RAPCAL}$ has been recently applied for the spectrospic determination of the electron density and temperature $[10,11]$. An example is given in Fig. 1 (right).

\section{ACKNOWLEDGMENTS}

This work has been supported by the Spanish Ministry of Science and Innovation under Research Grant No. ENE2008-06668-C02-02 and also by the Keep in touch Program of the European Union under Contract No. FU07-CT-2007-00050.

\section{REFERENCES}

1. R. Florido, R. Rodríguez, J. M. Gil, J. G. Rubiano, P. Martel, D. Suárez, M. Mendoza, and E. Mínguez, J. Phys.: Conf. Ser. 112, 042008 (2008), and references therein.

2. R. Rodríguez, R. Florido, J. M. Gil, J. G. Rubiano, P. Martel, and E. Mínguez, Laser Part. Beams (2008), doi:10.1017/S026303460800044X, and references therein.

3. M. F. Gu, Astrophys. J. 582, 1241-1250 (2003).

4. J. M. Gil, R. Rodríguez, R. Florido, J. G. Rubiano, P. Martel, and E. Mínguez, Laser Part. Beams 26, 21-31 (2008).

5. C. Bowen, R. W. Lee, and Y. Ralchenko, J. Quant. Spectrosc. Radiat. Transfer 99, 102-119 (2006).

6. J. G. Rubiano, R. Florido, C. Bowen, R. W. Lee, and Y. Ralchenko, High Energy Density Phys. 3, 225-232 (2007).

7. C. Chenais-Popovics, V. Malka, J. C. Gauthier, S. Gary, O. Peyrusse, M. Rabec-Le Gloahec, I. Matsushima, C. Bauche-Arnoult, A. Bachelier, and J. Bauche, Phys. Rev. E 65, 046418 (2002).

8. K. L. Wong, M. J. May, P. Beiersdorfer, K. B. Fournier, B. Wilson, G. V. Brown, and P. Springer, Phys. Rev. Lett. 90, 235001 (2003).

9. M. E. Foord, S. H. Glenzer, R. S. Thoe, K. L. Wong, K. B. Fournier, B. G. Wilson, and P. T. Springer, Phys. Rev. Lett. 85, 992-995 (2000).

10. R. Florido, T. Nagayama, R. C. Mancini, R. Tommasini, J. A. Delettrez, S. P. Regan, V. A. Smalyuk, R. Rodríguez, and J. M. Gil (2008), accepted for publication in Rev. Sci. Instrum.

11. J. M. Gil, R. Rodríguez, R. Florido, J. G. Rubiano, P. Martel, E. Mínguez, P. Sauvan, P. Angelo, R. Schott, E. Dalimier, and R. Mancini, "Spectrally resolved intensities of ultra-dense hot aluminum plasmas," 2008, these proceedings. 